\title{
Pre-analytical parameters associated with unsuccessful karyotyping in myeloid neoplasm: a study of 421 samples
}

\author{
M.F.M. Santos (id ${ }^{1}$, F.C.A.C. Oliveira (ii) ${ }^{1}$, R.K. Kishimoto (ii) ${ }^{1}$, D. Borri (ii) ${ }^{1}$, F.P.S. Santos (id) ${ }^{1}$, \\ P.V. Campregher (i) ${ }^{1}$, P.A.A. Silveira (i) ${ }^{1}$, N. Hamerschlak (i) ${ }^{1}$, C.L.P. Mangueira (ii) ${ }^{1}$, F.B. Duarte (i) ${ }^{2}$, \\ A.H. Crepaldi (ii) ${ }^{3}$, M.A. Salvino (ii) ${ }^{4}$ and E.D.R.P. Velloso (iD) ${ }^{1,5}$ \\ ${ }^{1}$ Hospital Israelita Albert Einstein, São Paulo, SP, Brasil \\ ${ }^{2}$ Hospital Universitário Walter Cantídio, Universidade Federal do Ceará, Fortaleza, CE, Brasil \\ ${ }^{3}$ Hospital de Câncer de Mato Grosso, Cuiabá, MT, Brasil \\ ${ }^{4}$ Hospital São Rafael/Monte Tabor, Salvador, BA, Brasil \\ ${ }^{5}$ Hospital das Clínicas, Faculdade de Medicina, Universidade de São Paulo, São Paulo, SP, Brasil
}

\begin{abstract}
Cytogenetics is essential in myeloid neoplasms (MN) and pre-analytical variables are important for karyotyping. We assessed the relationship between pre-analytical variables (time from collection to sample processing, material type, sample cellularity, and diagnosis) and failures of karyotyping. Bone marrow $(B M, n=352)$ and peripheral blood $(P B, n=69)$ samples were analyzed from acute myeloid leukemia $(n=113)$, myelodysplastic syndromes $(n=73)$, myelodysplastic syndromes/myeloproliferative neoplasms $(n=17)$, myeloproliferative neoplasms $(n=137)$, and other with conclusive diagnosis $(n=6)$, and reactive disorders/no conclusive diagnosis $(n=75)$. The rate of unsuccessful karyotyping was $18.5 \%$ and was associated with the use of $P B$ and a low number of nucleated cells $\left(\leqslant 7 \times 10^{3} / \mu \mathrm{L}\right)$ in the sample. High and low cellularity in BM and high and low cellularity in PB samples showed no metaphases in $3.9,39.7,41.9$, and $84.6 \%$ of cases, respectively. Collecting a good BM sample is the key for the success of karyotyping in $\mathrm{MN}$ and avoids the use of expensive molecular techniques.
\end{abstract}

Key words: Cytogenetics; Karyotype; Leukemia; Myelodysplasia; Myeloproliferative disease

\section{Introduction}

Cytogenetic study is essential for disease classification, prognostic assessment, and treatment (1). Typical chromosomal rearrangements are closely associated with specific tumor types, and the analysis of chromosomal abnormalities can be used to identify subpopulations that are most likely to benefit from specific treatments (specific drug targets) (2).

The World Health Organization recognizes genetic changes to define specific disease entities such as myeloid neoplasms (MN); myeloproliferative neoplasms (MPN); myelodysplastic/myeloproliferative neoplasms (MDS/MPN); myeloid and lymphoid neoplasms with PDGFR $\alpha, P D G F R \beta$ or FGRF1 or PMC1-JAK2 abnormalities (MLN); myelodysplastic syndromes (MDS); and acute myeloid leukemias (AML) (3).

Karyotyping (obtained from conventional cytogenetics) remains an essential test for myeloid malignancies. It allows a comprehensive structural analysis of the complete set of chromosomes, though only anomalies comprising more than $5 \mathrm{MB}$ can be detected. Chromosomes in metaphase are indispensable for karyotyping, and for this purpose, aspirated bone marrow or peripheral blood cells are cultured in vitro. The described rate of unsuccessful karyotyping in hematological malignancies samples is $10-20 \%$. Some pre-analytical variables such as the time from collection to processing, the sample type (bone marrow or peripheral blood), the volume, the nucleated cell number, and other conditions intrinsic to the processing methods (culture, harvesting, and banding) must be met for successful analysis (4).

This study aimed to assess the relationship between pre-analytical variables and failures associated with karyotyping in myeloid neoplasms (MN).

\section{Material and Methods}

\section{Case identification}

This study was approved by the Ethics Committee of Hospital Israelita Albert Einstein (No. 08942912.0.1001.0071), 
and written informed consent was obtained from all of the participants. This was a national multicenter study with participation of twelve centers from five (Northeastern, Midwest, and Southeast) Brazilian states, and 358 patients with suspicion or diagnosis of $\mathrm{MN}$ were included from October 2012 to September 2014. Diagnosis was based on each patient's clinical history, peripheral blood counts, hematopathology report, and results from flow cytometric immunophenotyping, and other molecular tests such as $B C R / A B L 1$ fusion, PDGFR $\alpha$ rearrangement, and JAK2 mutation. WHO criteria were used for the final diagnosis (3).

\section{Cytogenetic methods}

Bone marrow (BM, $n=352)$ and peripheral blood (PB, $\mathrm{N}=69$ ) samples were processed at the Cytogenetic Laboratory of Hospital Israelita Albert Einstein, São Paulo, Brazil. The transportation of samples from external centers was performed according to the standards and recommendations of the National Transportation Agency for biological materials.

Peripheral blood or bone marrow samples were cultured for 24 and $48 \mathrm{~h}$ without mitogenic agents and harvested following standard protocols (1).

\section{Pre-analytical variables}

The following pre-analytical variables were studied: 1) time from collection to sample processing (more or less than $24 \mathrm{~h}$ ); 2) material type (BM or PB); 3) sample cellularity ( $>7$ or $\leqslant 7 \times 10^{3} / \mu \mathrm{L}$ ), and 4 ) patient's diagnosis (AML, MDS, MPN, or MDS/MPN).

\section{Statistical analysis}

Chi-squared test or likelihood ratio test was used to verify associations of variables with karyotyping and notadjusted $\mathrm{OR}$ with $95 \% \mathrm{Cl}$ was estimated using bivariate logistic regression. Multivariate logistic regression was performed to adjust all variables in the model. The data were analyzed using IBM SPSS Statistics software (USA). The level of significance for the statistical tests was $5 \%$ $(P$ value $<0.05)$

\section{Results}

Three hundred and fifty-eight patients with a median age of 60 (16-86) years (52\% female) were analyzed; some patients had serial samples. From the 421 samples sent to the laboratory, $340(80.8 \%)$ samples belonged to patients with acute or chronic myeloid neoplasms with the following confirmed diagnoses: 1) 137 (32.5\%) MPN; 2) 113 (26.8\%) AML; 3) 73 (17.3\%) MDS; and 4) 17 (4.0\%) MDS/MPN. Most of the samples from the MPN group belonged to patients with myelofibrosis (MF, $n=58$ ), essential thrombocythemia $(n=41)$, polycythemia vera $(n=17)$, and non-categorized MPN $(n=21)$. The other samples belonged to patients with MLN with a PDGFR $\alpha$ rearrangement (1 case), lymphoid neoplasms (5 cases: $1 \mathrm{ALL}$,
$1 \mathrm{CLL}, 1$ hairy cell leukemia, and $2 \mathrm{NHL}$ ), or reactive disorders/non-conclusive final diagnoses (75 cases of thrombosis, polycythemia, eosinophilia, anemia).

Two hundred and eighty-two (67.0\%) samples were collected at the Hospital Israelita Albert Einstein, while $139(33.0 \%)$ samples came from 12 different centers. The median time from sample collection to culture processing was $24 \mathrm{~h}$ (306 samples in $24 \mathrm{~h}, 67$ in 24-48 h, 33 in 48-72 h, 15 in >72 h). In 94 cases, there were less than $7 \times 10^{3}$ nucleated cells per microliter of sample: 68 in BM and 26 in PB.

There were no evaluable metaphases (unsuccessful karyotyping - UK) in $78(18.5 \%)$ samples. The median number of metaphases analyzed was 20 (from 10 to 30) and only 23 cases presented less than 20 metaphases. There was no significant difference from samples processed in less than $24 \mathrm{~h}(52 / 306,17 \%$ of no metaphases), from 24 to $48 \mathrm{~h}(14 / 67,20.9 \%)$, and more than $48 \mathrm{~h}$ $(12 / 48,25 \%)$. The following pre-analytical variables were associated with UK: 1) peripheral blood as a sample $(P$ $<0.001) ; 2)$ low cellularity $\left(\leqslant 7 \times 10^{3} / \mu \mathrm{L}\right)$ samples $(\mathrm{P}<0.001)$, and 3 ) diagnosis $(P=0.018)$ (Supplementary Table S1). High and low cellularity in BM and high and low cellularity in PB samples showed no metaphases in 3.9, 39.7, 41.9, and $84.6 \%$ of cases, respectively. The diagnosis showed no significant differences based on the success of karyotyping when the "others" category was removed from the analysis. Most of the UK was recognized as related to low cellularity (49 samples $-62.8 \%$ ) or peripheral blood (40 samples $-51.3 \%$ ), although insufficient volumes (5 samples $-6.4 \%$ ) and aged samples (3 samples - $3.8 \%$ ) were also detected. Technical problems resulting in poor quality of metaphases were detected in $3(3.8 \%)$ cases. The multivariate analysis confirmed that only cellularity and type of sample were relevant $(P<0.001)$ for successful karyotyping in myeloid neoplasms.

\section{Discussion}

Cytogenetic studies are important for accurate diagnosis, appropriate treatment, and monitoring the response to therapy. Many of these aberrations have emerged as prognostic and predictive markers in hematologic cancers. Despite its importance, sometimes G-banding karyotyping cannot be performed due to technical difficulties such as low mitotic index and sample type collected.

The rate of unsuccessful karyotyping in this study was $18.5 \%$, similar to the described rate of UK in hematological malignancy (10-20\%) (4). In this study comprising only $\mathrm{MN}$, the UK was associated basically to the low number of nucleated cells and the PB samples.

Concentration of 1 million cells $/ 1 \mathrm{~mL}$ of medium is optimal, and most laboratories, including ours, attempt to obtain $1-2 \times 10^{3}$ cells $/ \mu \mathrm{L}\left(10^{6}\right.$ cells $\left./ \mathrm{mL}\right)$ culture, suspending the sample in 5-10 mL growth medium (5). Low cellularity was significantly associated with higher frequency 
of UK. If cell counts are low, guidelines $(5,6)$ suggest a culture of lower volume in order to maintain the cellular concentration. At least two different cultures are recommended, using two different media or two different culture times.

Although small or poor quality samples can sometimes fail to provide enough divisions, the high-count samples are most likely to fail completely. The vast majority of these cells are incapable of division, and their presence inhibits the few remaining cells that can divide (7). High cellularity BM sample showed UK in only $3.9 \%$, but in PB this rate was $41.9 \%$.

Non-evaluable metaphases in bone marrow samples were $10.8 \%(38 / 352)$ of cases. Some cases with UKs are undoubtedly due to insufficient cell number in the bone marrow aspirates, which is often the case in samples from AML with myelofibrosis and hypocellular AML. This result could possibly be remedied by using bone marrow biopsies (8). Despite this, in some circumstances, blood culture should be considered an alternative where a BM sample or culture has proved inadequate. Blood culture is not appropriate for all diagnoses, like MDS, MPD (except chronic granulocytic leukemia and myelofibrosis), or pancytopenic AML (9). For instance, aspiration of BM is often unsuccessful in MF, because of considerable fibrotic changes and replacement of hemopoietic cell clusters onto reticulin and collagen fibers (10). Peripheral blood specimens may yield informative results when the circulating blast cell percentage is higher than $10 \%$. In general, the abnormal clone can be identified in such specimens, albeit not as often as in bone marrow (6). On the other hand, bone marrow samples that have been contaminated with blood might not have an adequate number of spontaneously dividing cells. For this reason, it is important that the cytogenetics laboratory receive the first few milliliters of the bone marrow tap (11).

Peripheral blood samples were significantly associated with higher frequency of UK. Hussein et al. (12) reported successful karyotyping in $42 \%$ (102/242) of the cases of PB cytogenetics. In that study, differently from our findings, white blood cells did not independently predict the success of obtaining peripheral blood metaphases. Success was associated with an increasing level of myeloid progenitor cells or blasts of either myeloid or lymphoid lineage $(P=0.0007)$ and with abnormal $B M$ cytogenetics $(P=0.005)$ (12). A study of $B M$ and $P B$ samples from patients with MF with cytogenetic analysis of PB samples without stimulation of cell division was unsuccessful in all 10 patients due to either insufficient quantity or quality of metaphase plates, or lack of mitosis in the samples (10). However, Lozynskyy et al. (10) set up cell cultures of PB leukocytes stimulated in vitro with G-CSF and all 31 patients resulted in successful karyotyping. Moreover, chromosome abnormalities were detected in $19(45.2 \%)$ of the patients in cell cultures of
PB leukocytes stimulated in vitro with G-CSF, and in non-stimulated BM samples, abnormalities were detected in $19(45.2 \%)$ of all the patients, demonstrating that PB studies stimulated with appropriate mitogens may reduce the need of painful invasive diagnostic manipulations and facilitate follow-up of the patients.

Specimens should be received by the laboratory as soon as possible, without exposure to extreme temperatures, ideally within $24 \mathrm{~h}$, and the use of transport medium is strongly recommended to minimize drying-out of the sample and to maintain the viability of the cells $(6,9)$. There was no statistical significance regarding the time from collection to sample processing, all samples were sent to the laboratory in transport culture medium, and $88.6 \%$ set up cell cultures within $48 \mathrm{~h}$. In addition to time, other factors can influence the culture, such as sample type and cell concentration. A result can sometimes be obtained even from samples a few days old, with myeloid disorders being generally more tolerant of delay than samples from lymphoid disorders, and samples with a high white blood cell count usually need prompt attention (7). In this study, only a few "aged samples" were received; however, three of the UK cases were detected as "aged samples". Besides human errors in taking the bone marrow aspirates, such as volumes that are insufficient for performing at least two cellular cultures and diluting the bone marrow cells with peripheral blood, technical problems in the laboratory must be taken into account, such as equipment failure or preparation of reagents.

Diagnosis had no statistical impact on the success of karyotyping with the removal of the samples from the "other" category, so the diagnosis was not used in the final model. Our incidence of UK was 13.3 and $16.4 \%$ in AML and MDS, respectively. Incidence of an UK in AML is $10 \%$ in the literature and is related to poor prognosis $(P=0.002)(13)$. In MDS, UK is 6-7\%, mainly in patients with fibrotic or hypocellular marrows (14). In AML, UK occurs more commonly in older patients, predicts poor response to chemotherapy, and should be considered a high-risk feature (13). Study of FISH with MDS cases with G-banding failure did not identify abnormalities with poor prognosis and none of the patients had features of high risk MDS by morphologic criteria suggesting that this finding is associated with indolent forms of MDS (15). In contrast, in Cervera's study (16), unsuccessful conventional cytogenetic analysis in MDS was associated with worse survival compared to normal karyotyping.

Unsuccessful conventional cytogenetic analysis is directly related to cellularity and type of sample, so collecting a good and adequate sample is key for karyotyping success in myeloid neoplasm.

\section{Supplementary Material}

Click here to view [pdf]. 


\section{Acknowledgments}

The authors wish to thank the technical staff of the cytogenetic laboratory for their support, Isabel Clapis Bello and Sandra Saemi Nakashima for their administrative assistance, and Rogério Ruscitto do Prado for statistical analysis. We also thank centers that collaborated with samples, especially: Hospital São Rafael/ Monte Tabor-BA (Marco Aurelio Salvino, MD), Universidade Federal do Rio Grande do Norte - UFRN

\section{References}

1. Haferlach $\mathrm{C}$, Rieder $\mathrm{H}$, Lillington $\mathrm{DM}$, Dastugue $\mathrm{N}$, Hagemeijer A, Harbott J, et al. Proposals for standardized protocols for cytogenetic analyses of acute leukemias, chronic lymphocytic leukemia, chronic myeloid leukemia, chronic myeloproliferative disorders, and myelodysplastic syndromes. Genes Chromosomes Cancer 2007; 46: 494499, doi: 10.1002/gcc.20433.

2. Fröhling $\mathrm{S}$, Döhner $\mathrm{H}$. Chromosomal abnormalities in cancer. N Engl J Med 2008; 359: 722-734, doi: 10.1056/ NEJMra0803109.

3. Arber DA, Orazi A, Hasserjian R, Thiele J, Borowitz MJ, Le Beau MM, et al. The 2016 revision to the World Health Organization classification of myeloid neoplasms and acute leukemia. Blood 2016; 127: 2391-2405, doi: 10.1182/blood2016-03-643544.

4. Watson MS. Quality assurance and quality control in clinical cytogenetics. In: Dracopoli NC, Haines JL, Korf BR, Morton CC, Seidman CE, Seidman JG, Smith DR (Ed), Current Protocols in Human Genetics. New York: John Wiley; 2007. p 8.2.1-8.2.9.

5. Arsham MS, Barch MJ, Lawce HJ. The AGT Cytogenetics Laboratory Manual. Fourth edition. Hoboken, New Jersey: Wiley-Blackwell, 2017, doi: 10.1002/9781119061199.

6. Mikhail FM, Heerema NA, Rao KW, Burnside RD, Cherry AM, Cooley LD. Section E6.1-6.4 of the ACMG technical standards and guidelines: chromosome studies of neoplastic blood and bone marrow-acquired chromosomal abnormalities. Genet Med 2016; 18: 635-642, doi: 10.1038/gim.2016.50.

7. Swansbury J. Cytogenetic studies in hematologic malignancies: an overview. In: Swansbury J (Ed). Cancer Cytogenetics: methods and protocols. First edition. New Jersey: Humana press, 2003. p 13-17.

8. Al-Kali A, Konoplev S, Lin E, Kadia T, Faderl S, Ravandi F, et al. Hypocellular acute myeloid leukemia in adults: analysis of the clinical outcome of 123 patients. Haematologica 2012; 97: 235-40, doi: 10.3324/haematol.2011.046672.

9. Potter AM, Watmore A. Cytogenetics in myeloid leukaemia. In: Rooney DE and Czepulkowski BH (Ed). Human
(Rodolfo Soares, MD); Instituto de Assistência Médica ao Servidor Público Estadual - IAMSPE (Vera Lucia de Piratininga Figueiredo, MD), Faculdade de Medicina do ABC, São Paulo (Davimar Miranda Maciel Borducchi, MD), Universidade Federal do Ceará/Hospital Universitário Walter Cantidio (Fernando Barroso Duarte, MD), and Hospital de Câncer de Mato Grosso (Andre Henrique Crepaldi, MD). Funding was received from PROADI (25000.179520/2011-36, Ministério da Saúde-DECIT, Brazil).
Cytogenetics: A Practical Approach. Volume 2: Malignancy and Acquired Abnormalities. Second edition. Oxford: IRL Press at Oxford University Press, 1992. p 28.

10. Lozynskyy RY, Lozynska MR, Hontar YV, Huleyuk NL, Maslyak ZV, Novak VL. Study of cytogenetic abnormalities in G-CSF stimulated peripheral blood cells and non-stimulated bone marrow cells of patients with myelofibrosis. Exp Oncol 2016; 38: 40-44.

11. Gersen SL and Keagle MB (Ed). The principles of clinical cytogenetics. Second edition. New Jersey: Humana Press, 2005, doi: 10.1385/1592598331.

12. Hussein K, Ketterling RP, Hulshizer RL, Kuffel DG, Wiktor $A E$, Hanson CA, et al. Peripheral blood cytogenetic studies in hematological neoplasms: predictors of obtaining metaphases for analysis. Eur J Haematol 2008; 80: 318-321, doi: 10.1111/j.1600-0609.2007.01021.x.

13. Medeiros BC, Othus M, Estey EH, Fang M, Appelbaum FR. Unsuccessful diagnostic cytogenetic analysis is a poor prognostic feature in acute myeloid leukaemia. $\mathrm{Br} J$ Haematol 2014; 164: 245-250, doi: 10.1111/bjh.12625.

14. Stevens-Kroef MJ, Olde Weghuis D, Elldrissi-Zaynoun N, van der Reijden B, Cremers EMP, Alhan C, et al. Genomic array as compared to karyotyping in myelodysplastic syndromes in a prospective clinical trial. Genes Chromosomes Cancer 2017; 56: 524-534, doi: 10.1002/gcc.22 455.

15. Yang W, Stotler B, Sevilla DW, Emmons FN, Murty VV, Alobeid $\mathrm{B}$, et al. FISH analysis in addition to G-band karyotyping: utility in evaluation of myelodysplastic syndromes? Leuk Res 2010; 34: 420-425, doi: 10.1016/j. leukres.2009.09.013.

16. Cervera J, Solé F, Haase D, Luño E, Such E, Nomdedeu B, et al. Prognostic impact on survival of an unsuccessful conventional cytogenetic study in patients with myelodysplastic syndromes. In: Leukemia Research 2009; 33: Abstracts of the 10th International symposium on myelodysplastic syndromes, Patras (Greece) 6-9 May 2009. Abstract number: P030. 